\title{
Two-Channel Kondo Model as a Fixed Point of Local Electron-Phonon Coupling System
}

\author{
Hiroaki Kusunose and Kazumasa Mryake \\ Department of Material Physics, Faculty of Engineering Science, \\ Osaka University, Toyonaka 560
}

\begin{abstract}
It is shown on the basis of the multiplicative renormalization-group method of two-loop order that the low-energy effective Hamiltonian of a strongly coupled local electron-phonon system is mapped to the two-channel Kondo model. A phonon is treated as an Einstein oscillator with restricted Hilbert space such that up to one-phonon process is taken into account. By eliminating the high energy process of conduction electrons, it is shown that a certain class of couplings between ion vibrations and conduction electrons is selectively grown up. As a result the system is reduced to the two-channel Kondo model. The crossover temperature $T_{\mathrm{K}}$ and the renormalized phonon frequency $\Delta^{x}$ are expressed in terms of the mass ratio $m / M, m$ and $M$ being the mass of electron and ion, and the electron-phonon coupling $g / D, D$ being half the bandwidth of conduction electrons. The anomalous behaviors associated with this renormalization can be mesuarable if the condition $T_{\mathrm{K}}>\Delta^{x}$ is fulfilled. It is demonstrated that such condition is satisfied when $g / D$ is sufficiently large but in a realistic range.
\end{abstract}




\section{INTRODUCTION}

It has been recognized for these decades that a certain class of A15 compounds have puzzling physical properties [1,2]. One of the most important feature of these compounds is the large anharmonicity of ionic oscillations, which is exhibited, for example, as extremely small Debye-Waller factor at zero temperature limit [3] and anomalously large resistivity of the Ioffe-Regel limit at room temperature, where the mean free path of conduction electron is comparable to the lattice constant [2]. These compounds also show anomalously strong temperature dependence of electronic properties such as the magnetic susceptibility $\chi$ [ 4$]$, the Korringa constant $1 / T_{1} T\left(1 / T_{1}\right.$ being the nuclear relaxation rate) [5], and so on [2]. These anomalies can be explained by assuming an existence of extremely sharp peak in the density of states, of the order of $10 \sim 10^{2} \mathrm{~K}$, around the Fermi level. Such a sharp peak should arise from the many-body effect because the position of the peak always stays right at the Fermi

level independently of either components of compounds or degrees of stoichiometry [2].

Similar anomalies are exhibited in "heavy fermions" where the conduction electrons and an array of localized spins form a coherent quasiparticle band with extremely narrow width below the coherent temperature $T_{0}$. On the basis of this observation, the analogy between these two systems was pointed out [6,7]. In A15 compounds, the origin of the "spin" degrees of freedom is attributed to doubly degenerate stable positions of ion in a possible doublewell structure of ionic potential at $\mathrm{A}$ site of $\mathrm{A}_{3} \mathrm{~B}$ compounds [2]. Similar idea was proposed as a model for a layered compound $2 \mathrm{H}-\mathrm{TaSe}_{2}$ [8]. Such an anharmonicity of local-phonon can arise through strong electron-phonon coupling [2, 6,9], which causes screening of ionic potential the electron clouds feel and in turn decreases the restoring force for the ionic displacement leading to softening of ion oscillations. If the coupling is strong enough, a simple harmonic potential for the ion may deform into a double-well [2, 9].

In order that such system is renormalized into the Kondo model as in heavy fermions, we have to keep the electron-ion coupling containing at least quadratic term with respect to the ion displacement [6]. This is because it is such a term that gives the hopping between 
the two stable positions in the double-well corresponding to the spin-flip scattering in the Kondo problem which is indispensable to obtain the Kondo scaling. Along such a scenario, a crude and qualitative description of strongly coupled electron-phonon system was given previously [6].

The purpose of this paper is to investigate this problem more systematically without assuming the existense of double-well but on the model of an Einstein oscillator interacting with electron gas. By restricting the Hilbert space of phonon up to the one-phonon process, the Hamiltonian is transformed to the form which can be treated by the formalism of Vladár and Zawadowski [10]. It is shown that the Hamiltonian would be always renormalized into the form of Kondo model if the scaling step were not interrupted by the renormalized level splitting of ion oscillations which plays a role of an external pseudo-magnetic field acting on the pseudo-spin. In order that the "Kondo regime" is realized, the energy scale $T_{\mathrm{K}}$ characterizing the "Kondo renormalization" is larger than the renormalized level splitting $\Delta^{x}$. Then it is shown that the condition, $T_{\mathrm{K}}>\Delta^{x}$, is satisfied in the system with large but realistic value of electron-ion coupling.

In the region where the Kondo model is applicable, the effective Hamiltonian can be regarded as that of the two-channel Kondo model [11] [13] because the conduction electrons have the real-spin degrees of freedom other than that of the pseudo-spin which becomes explicit through the Vladár-Zawadowski renormalization. Here the real-spin degrees of freedom play a role of the channel there. It has been well recognized that the two-channel Kondo model exhibits the non-Fermi liquid fixed point in contrast with the single-channel Kondo model. Indeed, the two-channel model has been fully solved by variety of methods [14 20]. Quite recently, it has been shown that the susceptibility of real-spin, or channel, does exhibit the non-Fermi liquid behavior if the perturbations breaking the particle-hole symmetry, such as the Coulomb repulsion among conduction electrons or potential scattering, are taken into account [21]. This may potentially give an explanation for the anomalous temperature dependence of the spin susceptibility $\chi$ in A15 compound $\mathrm{V}_{3} \mathrm{Si}$ [ [4].

This paper is oraganized as follows. In $\S 2$ we present a local phonon model interacting 
with conduction electrons and derive a simplified model describing the low-energy physics. Next in $§ 3$, we derive the scaling equations following the argument by Vladár and Zawadowski [10] on the basis of the formalism of multiplicative renormalization-group [22, 23]. In $\S 4$ we solve the scaling equations and determine the crossover temperature $T_{\mathrm{K}}$ and the renormalized level splitting $\Delta^{x}$. On this basis we discuss the possibility of observing the anomaly associated with the two-channel Kondo model. In the final section, we summarize the results and discuss their implications, especially the relation with the so-called Migdal approximation which states the absence of the vertex correction for electron-phonon coupling and of the renormalization of the spin susceptiblility [24,25].

\section{MODEL HAMILTONIAN}

\section{A. Local Phonon Model}

We consider an Einstein oscillator interacting with conduction electrons. This simulates an optical phonon in A15 compounds where the transition metal ions maintain almost the atomic nature. In such a situation, an electrostatic restoring force tends to be screened by the cloud of electrons. This increases the anharmonicity of ionic oscillations and can make the adiabatic potential for ion displacement be even double-well like if the electron-phonon coupling is sufficiently strong. In order to verify such a scenario, we start with a Hamiltonian given as follows:

$$
\begin{aligned}
& H=H_{\mathrm{el}}+H_{\mathrm{ph}}+H_{\mathrm{ep}}, \\
& H_{\mathrm{el}}=\sum_{\mathbf{k}, \sigma} \xi_{\mathbf{k}} a_{\mathbf{k} \sigma}^{\dagger} a_{\mathbf{k} \sigma}, \\
& H_{\mathrm{ph}}=\Omega\left(b^{\dagger} b+\frac{1}{2}\right), \\
& H_{\mathrm{ep}}=\sum_{\mathbf{k k}^{\prime} \sigma} \int d \mathbf{r} v(\mathbf{r}-Q \hat{z}) \exp \left[i\left(\mathbf{k}^{\prime}-\mathbf{k}\right) \cdot \mathbf{r}\right] a_{\mathbf{k} \sigma}^{\dagger} a_{\mathbf{k}^{\prime} \sigma},
\end{aligned}
$$

where $a_{\mathbf{k}, \sigma}^{\dagger}$ and $b^{\dagger}$ denote the creation operator for the conduction electron with the wave vector $\mathbf{k}$ and the spin $\sigma$ and for the Einstein phonon with the energy $\Omega$, respectively. $\xi_{k}$ is 
the kinetic energy measured from the Fermi level.

The electrostatic potential $v$ in (4) is assumed to be contact type because its range is expected to be very short of the order of atomic radius as mentined above:

$$
v(\mathbf{r}-Q \hat{z}) \simeq-g \delta(\mathbf{r}-Q \hat{z})
$$

where $Q$ is the displacement of the ion along $z$-axis and $g$ is a coupling constant, which is positive and of the order of a bandwidth because its origin is the Coulomb attraction between electons and the ion. Thus the electron-phonon interaction (丑) is reduced to

$$
H_{\mathrm{ep}}=-g \sum_{\mathbf{k}, \mathbf{k}^{\prime}} \sum_{\sigma} \exp \left[i\left(k_{z}^{\prime}-k_{z}\right) Q\right] a_{\mathbf{k} \sigma}^{\dagger} a_{\mathbf{k}^{\prime} \sigma} .
$$

\section{B. Simplification of the Model}

As it will be shown below, the interaction between ionic vibrations and electrons near the Fermi level increases logarithmically as eliminating high-energy processes. Namely, the electrons in the vicinity of the Fermi level is crucial; so that the polarization of conduction electrons, which is expressed in terms of directional dependence of the wave vector $\mathbf{k}$ near the Fermi level, plays a crucial role. Therefore, we first introduce the spherical wave representation for the creation operator of conduction electrons as follows:

$$
a_{k l m \sigma}^{\dagger}=(-i)^{l} \frac{k R}{\sqrt{6 \pi}} \int d \hat{k} Y_{l m}(\hat{k}) a_{\mathbf{k}, \sigma}^{\dagger}
$$

where $Y_{l m}$ is the spherical harmonics and $R$ denotes the radius of the system. With the use of a linearized dispersion for conduction electrons, $\xi_{k} \sim k_{\mathrm{F}}\left(k-k_{\mathrm{F}}\right) / m, k_{\mathrm{F}}$ being the Fermi wavenumber and $m$ the mass of conduction electron, $H_{\mathrm{el}}$ given by (2) is reduced to

$$
H_{\mathrm{el}}=D \sum_{l m \sigma} \int_{-1}^{1} d k k a_{k l m \sigma}^{\dagger} a_{k l m \sigma},
$$

where the bandwidth $D$ of conduction electrons is given as

$$
D=\frac{k_{\mathrm{F}}^{3} R}{m \pi}
$$


Secondly, we make a simplification of the phonon part $H_{\mathrm{ph}}$, (3). The displacement $Q$ and its canonical momentum $P$ are represented in terms of the phonon operators, $b^{\dagger}$ and $b$, as follows:

$$
\begin{aligned}
& Q=q\left(b+b^{\dagger}\right), \\
& P=\frac{1}{2 q i}\left(b-b^{\dagger}\right),
\end{aligned}
$$

where $q \equiv \sqrt{1 / 2 M \Omega}, M$ being the mass of the ion. Since the low-energy phonon states are important, we restrict the Hilbert space of the phonon in such a way that only the states with $n=<b^{\dagger} b>=0$ or 1 are included in the low-energy effective (fixed point) Hamiltonian. Then, $Q$, (10), and $P$, (11), are represented in this restricted Hilbert space as

$$
\begin{aligned}
& Q=q \sum_{n n^{\prime}}^{0,1} b_{n}^{\dagger} \tau_{n n^{\prime}}^{x} b_{n^{\prime}}, \quad Q^{2}=q^{2} \sum_{n n^{\prime}}^{0,1} b_{n}^{\dagger}\left(2 \delta_{n n^{\prime}}-\tau_{n n^{\prime}}^{z}\right) b_{n^{\prime}} \\
& P=\frac{1}{2 q} \sum_{n n^{\prime}}^{0,1} b_{n}^{\dagger} \tau_{n n^{\prime}}^{y} b_{n^{\prime}}, \quad P^{2}=\frac{1}{4 q^{2}} \sum_{n n^{\prime}}^{0,1} b_{n}^{\dagger}\left(2 \delta_{n n^{\prime}}-\tau_{n n^{\prime}}^{z}\right) b_{n^{\prime}}
\end{aligned}
$$

where $\tau^{i}(i=x, y, z)$ is the $i$-th component of the Pauli operator, and $b_{n}^{\dagger}$ is the pseudofermion operator creating the $n$-phonon state so that $\frac{1}{2} \tau$ can be regarded as the pseudo-spin corresponding to the phonon degrees of freedom.

It is noted that $(Q)^{2} \neq\left(Q^{2}\right)$ and $(P)^{2} \neq\left(P^{2}\right)$ since $\left(Q^{2}\right)$ and $\left(P^{2}\right)$ include virtual 2-phonon process. Indeed, the matrix elements of $Q^{2}$ are given as follows:

$$
\begin{aligned}
& <0\left|Q^{2}\right| 0>=<0|Q| 1><1|Q| 0>=q^{2} \\
& <1\left|Q^{2}\right| 1>=<1|Q| 0><0|Q| 1>+<1|Q| 2><2|Q| 1>=\frac{3}{2} q^{2} \\
& <1\left|Q^{2}\right| 0>=<0\left|Q^{2}\right| 1>=0
\end{aligned}
$$

where $\mid n>$ denotes the $n$-phonon state. These matrix elements are equivalent to the expression (12). One can show that the same arguments hold for the operator $P^{2}$.

For the later discussions, it is more convenient to introduce the alternative basis for the representation of phonon states as follows:

$$
b_{\uparrow}^{\dagger}=\frac{1}{\sqrt{2}}\left(b_{0}^{\dagger}-b_{1}^{\dagger}\right), \quad b_{\downarrow}^{\dagger}=\frac{-1}{\sqrt{2}}\left(b_{0}^{\dagger}+b_{1}^{\dagger}\right) .
$$


Then the non-vanishing matrix elements of $Q$ are given as

$$
<\uparrow|Q| \uparrow>=-q, \quad<\downarrow|Q| \downarrow>=+q,
$$

where $\mid \uparrow>$ denotes the state $b_{\uparrow}^{\dagger}|\operatorname{vac}>,| \operatorname{vac}>$ being the vacuum state, and so on.

In this representation, Eqs. (12) and (13) are transformed as

$$
\begin{aligned}
& Q=-q \sum_{\alpha \beta}^{\uparrow, \downarrow} b_{\alpha}^{\dagger} \tau_{\alpha \beta}^{z} b_{\beta}, \quad Q^{2}=q^{2} \sum_{\alpha \beta}^{\uparrow, \downarrow} b_{\alpha}^{\dagger}\left(2 \delta_{\alpha \beta}+\tau_{\alpha \beta}^{x}\right) b_{\beta}, \\
& P=\frac{1}{2 q} \sum_{\alpha \beta}^{\uparrow, \downarrow} b_{\alpha}^{\dagger} \tau_{\alpha \beta}^{y} b_{\beta}, \quad P^{2}=\frac{1}{4 q^{2}} \sum_{\alpha \beta}^{\uparrow, \downarrow} b_{\alpha}^{\dagger}\left(2 \delta_{\alpha \beta}+\tau_{\alpha \beta}^{x}\right) b_{\beta},
\end{aligned}
$$

and $H_{\mathrm{ph}}$, (3), can be written in the restricted Hilbert space as

$$
H_{\mathrm{ph}}=\frac{1}{2} \Omega \sum_{\alpha \beta}^{\uparrow, \downarrow} b_{\alpha}^{\dagger} \tau_{\alpha \beta}^{x} b_{\beta}+\Omega .
$$

Thirdly, we simplify the electron-phonon interaction $H_{\mathrm{ep}}$, (4). Since the exponent of $\exp \left[i\left(\mathbf{k}^{\prime}-\mathbf{k}\right) \cdot \mathbf{r}\right]$ in (4) is of the order of $k_{\mathrm{F}} Q \sim k_{\mathrm{F}} q \sim(m / M)^{1 / 4} \ll 1$, we expand the exponential with respect to $k_{\mathrm{F}} Q$ up to second order. Then, using the expressions (19), we obtain

$$
\begin{aligned}
H_{\mathrm{ep}} & =-g \sum_{\mathbf{k} \mathbf{k}^{\prime}} \sum_{\sigma} \sum_{\alpha \beta} a_{\mathbf{k} \sigma}^{\dagger} a_{\mathbf{k}^{\prime} \sigma}\left[\sum_{i} V_{\hat{k} \hat{k}^{\prime}}^{i} b_{\alpha}^{\dagger} \tau_{\alpha \beta}^{i} b_{\beta}+V_{\hat{k} \hat{k}^{\prime}}^{0} \delta_{\alpha \beta}\right]+\mathcal{O}\left(\left(k_{\mathrm{F}} Q\right)^{3}\right), \\
V_{\hat{k} \hat{k}^{\prime}}^{x} & =-\frac{1}{2}\left(\hat{k_{z}^{\prime}}-\hat{k_{z}}\right)^{2} k_{\mathrm{F}}^{2} q^{2}, \quad V_{\hat{k} \hat{k}^{\prime}}^{y}=0, \quad V_{\hat{k} \hat{k}^{\prime}}^{z}=-i\left(\hat{k_{z}^{\prime}}-\hat{k_{z}}\right) k_{\mathrm{F}} q, \\
V_{\hat{k} \hat{k^{\prime}}}^{0} & =1-\left(\hat{k_{z}^{\prime}}-\hat{k_{z}}\right)^{2} k_{\mathrm{F}}^{2} q^{2} .
\end{aligned}
$$

It is noted that the interaction $V_{\hat{k} \hat{k}^{\prime}}^{x}$, (23), arises from $Q^{2}$-term and gives the pseudo-spin flip scattering which is the heart of the Kondo effect [26].

In order to treat the problem in the spherical representation, we introduce the interaction matrices $V_{l l^{\prime}}^{i}$ defined by

$$
V_{\hat{k} \hat{k}^{\prime}}^{i}=4 \pi \sum_{l l^{\prime}} i^{l^{\prime}-l} Y_{l 0}(\hat{k}) Y_{l^{\prime} 0}^{*}\left(\hat{k}^{\prime}\right) V_{l l^{\prime}}^{i}
$$

It is noted that the phonon vibration along the $z$-axis interacts only with the component of $m=0$. It is convenient to introduce the basis of conduction electron corresponding to those of the phonon, (17), as follows: 


$$
\begin{aligned}
& a_{k \uparrow \sigma}^{\dagger}=\frac{1}{\sqrt{2}}\left(a_{k 00 \sigma}^{\dagger}+a_{k 10 \sigma}^{\dagger}\right), \\
& a_{k \downarrow \sigma}^{\dagger}=\frac{1}{\sqrt{2}}\left(a_{k 00 \sigma}^{\dagger}-a_{k 10 \sigma}^{\dagger}\right), \\
& a_{k d \sigma}^{\dagger}=a_{k 20 \sigma}^{\dagger} .
\end{aligned}
$$

With the use of (7) and (25), the simplified Hamiltonian is given as

$$
\begin{aligned}
H / D & =H_{\mathrm{el}}+H_{\mathrm{ph}}+H_{\mathrm{ep}}, \\
H_{\mathrm{el}} & =\sum_{\sigma} \sum_{l}^{\uparrow, \downarrow, d} \int_{-1}^{1} d k k a_{k l \sigma}^{\dagger} a_{k l \sigma}, \\
H_{\mathrm{ph}} & =\sum_{i}^{x, y, z} \sum_{\alpha \beta}^{\uparrow, \downarrow} \Delta^{i} b_{\alpha}^{\dagger} \tau_{\alpha \beta}^{i} b_{\beta}, \\
H_{\mathrm{ep}} & =\int_{-1}^{1} d k \int_{-1}^{1} d k^{\prime} \sum_{\sigma} \sum_{l l^{\prime}}^{\uparrow, \downarrow, d} \sum_{\alpha \beta}^{\uparrow, \downarrow} \sum_{i}^{x, y, z} a_{k l \sigma}^{\dagger} v_{l l^{\prime}}^{i} a_{k^{\prime} l^{\prime} \sigma} b_{\alpha}^{\dagger} \tau_{\alpha \beta}^{i} b_{\beta},
\end{aligned}
$$

where $v_{l l^{\prime}}^{i} \equiv-g V_{l l^{\prime}}^{i}$ are the electron-phonon couplings which are non-dimensional and $\Delta^{i}$ is a fictitious magnetic field acting on the pseudo-spin $\frac{1}{2} \tau$. The explicit forms of $v_{l l^{\prime}}^{i}$ and $\Delta^{i}$ in the new basis are given as follows:

$$
\begin{aligned}
& \hat{v}^{x}=\frac{1}{3} \frac{g}{D} k_{\mathrm{F}}^{2} q^{2}\left(\begin{array}{ccc}
0 & 1 & -1 / \sqrt{10} \\
1 & 0 & -1 / \sqrt{10} \\
-1 / \sqrt{10} & -1 / \sqrt{10} & 0
\end{array}\right), \\
& \hat{v}^{y}=0, \quad \hat{v}^{z}=\frac{1}{\sqrt{3}} \frac{g}{D} k_{\mathrm{F}} q\left(\begin{array}{ccc}
1 & 0 & 0 \\
0 & -1 & 0 \\
0 & 0 & 0
\end{array}\right) \\
& \Delta^{x}=\frac{1}{2} \frac{\Omega}{D}, \quad \Delta^{y}=\Delta^{z}=0 .
\end{aligned}
$$

It is noted that the pseudo-spin $\tau$ appears in the Hamiltonian (29) representing the phonon degree of freedom, while it does not in the electronic degrees of freedom at the biginning but it is induced by the renormalization group evolution as discussed below. It is also noted that the potential scattering is neglected in (29), although it has been recently suggested that the potential scattering is relevant to discuss the magnetic property on the basis of the pseudo-spin model [21. 


\section{SCALING EQUATIONS}

The Hamiltonian (29) involves the logarithmic divergence at the Fermi level due to the screening of local internal degrees of freedom by the conduction electons as discussed by Vladár and Zawadowski [10]. Since we are intersted in the physics near the Fermi level, we eliminate the high energy process in the sense of the renormalization group evolution. To this end, we apply the multiplicative renormalization-group [22,23] formalism with help of the Abrikosov pseudo-fermion representation of the pseudo-spin [27].

\section{A. Multiplicative Renormalization-Group Transformation}

First, we discuss the formalism for the multiplicative Renormalization-group transformation on the basis of the perturbation theory.

It is useful to introduce the Matsubara Green functions for the electron and the pseudofermion defined as

$$
G=\frac{1}{i \omega-\xi_{k}}
$$

and

$$
\mathcal{G}_{\alpha \beta}=\frac{1}{i \epsilon-\lambda-\sum_{i} \Delta^{i} \tau_{\alpha \beta}^{i}-\Sigma_{\alpha \beta}},
$$

respectively, where $\omega$ and $\epsilon$ denote the Matsubara frequency. The electron self-energy contains a closed pseudo-fermion loop and it tends to zero as $\lambda \rightarrow \infty$; thus, the electron selfenergy is ignored. It is noted that $\mathcal{G}$ and the self-energy $\Sigma$ for pseudo-fermion are matrices in the pseudo-spin space.

It is assumed that the system with a reduced bandwidth $D^{\prime}$ behaves at low energies in a way similar to that of original system with the bandwidth $D$, if the couplings $v_{l l^{\prime}}^{i}$ and the

phonon energy $\Delta^{i}$ are modified apropriately. The difference between the original and the scaled Green function and the vertex function can be given by multiplicative factors $Z_{2}$ and 
$Z_{l l^{\prime}}^{i}$, respectively. The multiplicative factor for the conduction electron is given as $Z_{1}=1$. The multiplicative renormalization-group transformation can be given by

$$
\begin{aligned}
& \mathcal{G}_{\alpha \beta}\left(\frac{\omega}{D^{\prime}}, v_{l l^{\prime}}^{\prime i}, \Delta^{\prime i}\right)=Z_{2}\left(\frac{D^{\prime}}{D}, v_{l l^{\prime}}^{i}\right) \mathcal{G}_{\alpha \beta}\left(\frac{\omega}{D}, v_{l l^{\prime}}^{i}, \Delta^{i}\right), \\
& \gamma_{l l^{\prime}}^{i}\left(\frac{\omega}{D^{\prime}}, v_{l l^{\prime}}^{\prime i}\right)=\left[Z_{l l^{\prime}}^{i}\left(\frac{D^{\prime}}{D}, v_{l l^{\prime}}^{i}\right)\right]^{-1} \gamma_{l l^{\prime}}^{i}\left(\frac{\omega}{D}, v_{l l^{\prime}}^{i}\right),
\end{aligned}
$$

and

$$
v_{l l^{\prime}}^{i i}=Z_{2}^{-1} Z_{l l^{\prime}}^{i} v_{l l^{\prime}}^{i},
$$

where $\gamma_{l l^{\prime}}^{i}$ is the normalized vertex related to the vertex $\Gamma_{l l^{\prime}}^{i}$ as

$$
\Gamma_{l l^{\prime}}^{i}=\gamma_{l l^{\prime}}^{i} v_{l l^{\prime}}^{i}
$$

The new scaled couplings $v_{l l^{\prime}}^{\prime i}$ and parameters $\Delta^{\prime i}$ are labeled by prime. An alternative multiplicative renormalization relation for the vertex is given by multiplying Eq. (39) by $v_{l l^{\prime}}^{\prime i}$ and by inserting the Eq. (40) and it is obtained as

$$
\Gamma_{l l^{\prime}}^{i}\left(\frac{\omega}{D^{\prime}}, v_{l l^{\prime}}^{i}\right)=Z_{2}^{-1}\left(\frac{D^{\prime}}{D}, v_{l l^{\prime}}^{i}\right) \Gamma_{l l^{\prime}}^{i}\left(\frac{\omega}{D}, v_{l l^{\prime}}^{i}\right) .
$$

The multiplicative factor for $\Delta$ is not simple and it will be given only along the detailed calculation. It is important to note that the multiplicative factors depend only on the relative change of the bandwidth $D^{\prime} / D$ and on the couplings $v_{l l^{\prime}}^{i}$.

In order to construct the renormalization-group transformations given by Eqs. (38) (40), the perturbation theory is applied. Therefore the result to be derived is not valid in the region of strong coupling. According to the perturbation theory, the scaling equations is given as

$$
\frac{d}{d x} v_{l l^{\prime}}^{i}=\beta\left(v_{l l^{\prime}}^{i}\right)
$$

and

$$
\frac{d}{d x} \Delta^{i}=f\left(v_{l l^{\prime}}^{i}, \Delta^{i}\right),
$$

where $x=\ln \left(D / D^{\prime}\right)$. The irrelevant part of the phase space for the conduction electrons will be eliminated by integrating Eqs. (43) and (44), but the procedure can be applied only as far as $x<\min \left[\ln \left(D / k_{\mathrm{B}} T\right), \ln \left(1 /\left|\boldsymbol{\Delta}^{\prime}\right|\right)\right]$. 


\section{B. Derivation of Scaling Equations}

In order to construct the scaling equations, it is convenient to use a new basis giving $\bar{\Delta}^{z} \neq 0$, which is obtained by the rotation by the angle $\pi / 2$ around the $y$-axis, instead of the basis where $\Delta^{x} \neq 0$ and $\bar{\Delta}^{z}=0$. In this new rotated representation, the couplings and the fictitious magnetic field are related as $v^{x}=\bar{v}^{z}, v^{y}=\bar{v}^{y}, v^{z}=-\bar{v}^{x}$, and $\Delta^{x}=\bar{\Delta}^{z}, \Delta^{y}=\bar{\Delta}^{y}$, $\Delta^{z}=-\bar{\Delta}^{x}$, respectively.

The vertex corrections of first and second order are shown in Fig. 1(a), 1(b) and Fig. 2, respectively. Their analytical expressions are given as follows:

$$
\Gamma_{l l^{\prime}}^{i(\mathrm{I})}=-2 i \sum_{j k}\left(\bar{v}^{j} \bar{v}^{k}\right)_{l l^{\prime}} \epsilon^{i j k} \ln \frac{D}{|\omega|}
$$

and

$$
\Gamma_{l l^{\prime}}^{i(\mathrm{II})}=n \sum_{j}\left[2 \operatorname{Tr}\left(\bar{v}^{i} \bar{v}^{j}\right) \bar{v}_{l l^{\prime}}^{j}-\operatorname{Tr}\left(\bar{v}^{j} \bar{v}^{j}\right) \bar{v}_{l l^{\prime}}^{i}\right] \ln \frac{D}{|\omega|}
$$

where $n$ represents a degree of freedom of spins of the conduction electron, i.e. $n=2$.

The self-energy shown in Fig. 3 is given by

$$
\Sigma_{\alpha \beta}^{(\mathrm{I})}=-n\left[\omega \sum_{i} \operatorname{Tr}\left(\bar{v}^{i} \bar{v}^{i}\right) \delta_{\alpha \beta}-\sum_{i j}\left\{2 \operatorname{Tr}\left(\bar{v}^{i} \bar{v}^{j}\right) \bar{\Delta}^{i}-\operatorname{Tr}\left(\bar{v}^{i} \bar{v}^{i}\right) \bar{\Delta}^{j}\right\} \bar{\tau}_{\alpha \beta}^{j}\right] \ln \frac{D}{|\omega|} .
$$

It is noted that the self-energy contains off-diagonal terms which are proportional to $\bar{\tau}_{\alpha \beta}^{x}$ and $\bar{\tau}_{\alpha \beta}^{y}$. Let us define $\bar{\Delta}^{i}$ such that the renormalization factor $Z_{2}$ is independent of $\bar{\Delta}^{i}$. Explicit expresssions of $\bar{\Delta}^{i}$ and $Z_{2}$ are given by

$$
\begin{aligned}
& \bar{\Delta}^{\prime i}=\bar{\Delta}^{i}+2 n \sum_{j}\left[\operatorname{Tr}\left(\bar{v}^{i} \bar{v}^{j}\right) \bar{\Delta}^{j}-\operatorname{Tr}\left(\bar{v}^{j} \bar{v}^{j}\right) \bar{\Delta}^{i}\right] \ln \frac{D}{D^{\prime}} \\
& Z_{2}=1+n \sum_{i} \operatorname{Tr}\left(\bar{v}^{i} \bar{v}^{i}\right) \ln \frac{D}{D^{\prime}}
\end{aligned}
$$

It will be shown later that $\bar{\Delta}^{x}$ and $\bar{\Delta}^{y}$ can always be eliminated by a rotation around the $y$-axis and $x$-axis in the pseudo-spin space. In the case $\bar{\Delta}^{i}=\bar{\Delta}^{z} \delta_{i z}$, Eqs. (47) and (48) are rewritten as 


$$
\begin{aligned}
\Sigma_{\alpha \beta}^{(\mathrm{I})} & =-n\left[\omega \sum_{i} \operatorname{Tr}\left(\bar{v}^{i} \bar{v}^{i}\right) \delta_{\alpha \beta}-2 \bar{\Delta}^{z} \operatorname{Tr}\left(\bar{v}^{x} \bar{v}^{z}\right) \bar{\tau}_{\alpha \beta}^{x}\right. \\
- & \left.2 \bar{\Delta}^{z} \operatorname{Tr}\left(\bar{v}^{y} \bar{v}^{z}\right) \bar{\tau}_{\alpha \beta}^{y}-\bar{\Delta}^{z} \operatorname{Tr}\left(\bar{v}^{z} \bar{v}^{z}-\bar{v}^{x} \bar{v}^{x}-\bar{v}^{y} \bar{v}^{y}\right) \bar{\tau}_{\alpha \beta}^{z}\right] \ln \frac{D}{|\omega|}, \\
\bar{\Delta}^{\prime} & =2 n \bar{\Delta}^{i} \operatorname{Tr}\left(\bar{v}^{i} \bar{v}^{z}\right) \ln \frac{D}{D^{\prime}}, \quad(i=x, y), \\
\bar{\Delta}^{\prime z} & =\bar{\Delta}^{z}\left[1-2 n \operatorname{Tr}\left(\bar{v}^{x} \bar{v}^{x}+\bar{v}^{y} \bar{v}^{y}\right) \ln \frac{D}{D^{\prime}}\right],
\end{aligned}
$$

respectively.

The renormalized coupling ${\overline{v^{\prime}}}_{l l^{\prime}}^{i}$ is obtained by Eqs. (42), (45), (46) and (49) as follows:

$$
{\overline{v^{\prime}}}_{l l^{\prime}}^{i}=\bar{v}_{l l^{\prime}}^{i}-2 i \sum_{j k}\left(\bar{v}^{j} \bar{v}^{k}\right)_{l l^{\prime}} \epsilon^{i j k} \ln \frac{D}{D^{\prime}}-2 n \sum_{j}\left[\operatorname{Tr}\left(\bar{v}^{j} \bar{v}^{j}\right) \bar{v}_{l l^{\prime}}^{i}-\operatorname{Tr}\left(\bar{v}^{i} \bar{v}^{j}\right) \bar{v}_{l l^{\prime}}^{j}\right] \ln \frac{D}{D^{\prime}}
$$

Although the renormalization-group transformation generates the parameters $\bar{\Delta}^{\prime x}$ and $\bar{\Delta}^{\prime y}$, they can be eliminated by the rotation around the $x$ - and $y$-axes. The angles of the rotations are

$$
\begin{aligned}
& \alpha_{x}=2 n \operatorname{Tr}\left(\bar{v}^{y} \bar{v}^{z}\right) \ln \frac{D}{D^{\prime}}, \\
& \alpha_{y}=2 n \operatorname{Tr}\left(\bar{v}^{x} \bar{v}^{z}\right) \ln \frac{D}{D^{\prime}} .
\end{aligned}
$$

Then, $\bar{\Delta}^{\prime} i$ is transformed to

$$
\begin{aligned}
& {\overline{\Delta^{\prime \prime}}}^{x}={\overline{\Delta^{\prime \prime}}}^{y}=0, \\
& {\overline{\Delta^{\prime \prime}}}^{z}=\bar{\Delta}^{z}\left[1-2 n \operatorname{Tr}\left(\bar{v}^{x} \bar{v}^{x}+\bar{v}^{y} \bar{v}^{y}\right) \ln \frac{D}{D^{\prime}}\right] .
\end{aligned}
$$

Furthermore, these rotations modify the couplings ${\overline{v^{\prime}}}_{l l^{\prime}}^{i}$ to ${\overline{v^{\prime \prime}}}_{l l^{\prime}}^{i}$,

$$
\begin{aligned}
& {\overline{v^{\prime \prime}}}_{l l^{\prime}}^{i}={\overline{v^{\prime}}}_{l l^{\prime}}^{i}-2 n \operatorname{Tr}\left(\bar{v}^{i} \bar{v}^{z}\right) \bar{v}_{l l^{\prime}}^{z} \ln \frac{D}{D^{\prime}}, \quad(i=x, y), \\
& {\overline{v^{\prime \prime}}}_{l l^{\prime}}^{z}=\bar{v}_{l l^{\prime}}^{z}+2 n\left[\operatorname{Tr}\left(\bar{v}^{x} \bar{v}^{z}\right) \bar{v}_{l l^{\prime}}^{x}+\operatorname{Tr}\left(\bar{v}^{y} \bar{v}^{z}\right) \bar{v}_{l l^{\prime}}^{y}\right] \ln \frac{D}{D^{\prime}}
\end{aligned}
$$

Combining the relations (53), (58), and (59), we obtain the scaling equations for the coupling $\bar{v}$ 's as

$$
\frac{d}{d x} \bar{v}_{l l^{\prime}}^{i}=-2 i \sum_{j k} \epsilon^{i j k}\left(\bar{v}^{j} \bar{v}^{k}\right)_{l l^{\prime}}-2 n\left[\sum_{j}\left\{\operatorname{Tr}\left(\bar{v}^{j} \bar{v}^{j}\right) \bar{v}_{l l^{\prime}}^{i}-\operatorname{Tr}\left(\bar{v}^{i} \bar{v}^{j}\right) \bar{v}_{l l^{\prime}}^{j}\right\}\right.
$$




$$
\begin{aligned}
& \left.\quad+\operatorname{Tr}\left(\bar{v}^{i} \bar{v}^{z}\right) \bar{v}_{l l^{\prime}}^{z}\right], \quad(i=x, y), \\
& \frac{d}{d x} \bar{v}_{l l^{\prime}}^{z}=-2 i \sum_{j k} \epsilon^{z j k}\left(\bar{v}^{j} \bar{v}^{k}\right)_{l l^{\prime}}-2 n\left[\sum_{j}\left\{\operatorname{Tr}\left(\bar{v}^{j} \bar{v}^{j}\right) \bar{v}_{l l^{\prime}}^{z}-\operatorname{Tr}\left(\bar{v}^{z} \bar{v}^{j}\right) \bar{v}_{l l^{\prime}}^{j}\right\}\right. \\
& \left.\quad-\operatorname{Tr}\left(\bar{v}^{x} \bar{v}^{z}\right) \bar{v}_{l l^{\prime}}^{x}-\operatorname{Tr}\left(\bar{v}^{y} \bar{v}^{z}\right) \bar{v}_{l l^{\prime}}^{y}\right],
\end{aligned}
$$

and the scaling equation for the fictitious magnetic field $\bar{\Delta}^{z}$ as

$$
\frac{d}{d x} \bar{\Delta}^{z}=-2 n \operatorname{Tr}\left(\bar{v}^{x} \bar{v}^{x}+\bar{v}^{y} \bar{v}^{y}\right) \bar{\Delta}^{z} .
$$

These scaling equations can be represented in the original basis of pseudo-spin, i.e., in terms of $v$ 's and $\Delta$ 's without bar, if the pseudo-spin axis is rotated back around the $y$-axis by the angle $-\pi / 2$. Then, the scaling equations are given as follows:

$$
\begin{aligned}
& \frac{d}{d x} v_{l l^{\prime}}^{x}=-2 i \sum_{j k} \epsilon^{x j k}\left(v^{j} v^{k}\right)_{l l^{\prime}}-2 n\left[\sum_{j}\left\{\operatorname{Tr}\left(v^{j} v^{j}\right) v_{l l^{\prime}}^{x}-\operatorname{Tr}\left(v^{x} v^{j}\right) v_{l l^{\prime}}^{j}\right\}\right. \\
& \left.\quad-\operatorname{Tr}\left(v^{x} v^{z}\right) v_{l l^{\prime}}^{z}-\operatorname{Tr}\left(v^{x} v^{y}\right) v_{l l^{\prime}}^{y}\right], \\
& \frac{d}{d x} v_{l l^{\prime}}^{i}=-2 i \sum_{j k} \epsilon^{i j k}\left(v^{j} v^{k}\right) l l^{l^{\prime}}-2 n\left[\sum_{j}\left\{\operatorname{Tr}\left(v^{j} v^{j}\right) v_{l l^{\prime}}^{i}-\operatorname{Tr}\left(v^{i} v^{j}\right) v_{l l^{\prime}}^{j}\right\}\right. \\
& \left.\quad+\operatorname{Tr}\left(v^{i} v^{x}\right) v_{l l^{\prime}}^{x}\right], \quad(i=y, z), \\
& \frac{d}{d x} \Delta^{x}=-2 n \operatorname{Tr}\left(v^{y} v^{y}+v^{z} v^{z}\right) \Delta^{x} .
\end{aligned}
$$

\section{RENORMALIZATION-GROUP EVOLUTIONS}

In this section, solving Eqs. (63)-(65), we determine the characteristic temperature $T_{\mathrm{K}}$, which characterizes the crossover between weak and strong coupling regime, and the renormalized first excited energy of phonon $\Delta^{x}(x)$, below which the the renormalization-group transformation cannot be proceeded further. Then, the typical cases of interest will be discussed. 


\section{A. Solution of Scaling Equations}

First, we discuss the case $\left|v_{l l^{\prime}}^{x}\right|,\left|v_{l l^{\prime}}^{y}\right| \ll v_{l l^{\prime}}^{z}$, which appears at the initial stage of renormalization-group evolution of the present problem. In this case, we can linearize the scaling equations (63) and (64) with respect to $v_{l l^{\prime}}^{x}$ and $v_{l l^{\prime}}^{y}$. Then, the linearized version of the scaling equations, (63) and (64), are given as follows:

$$
\begin{aligned}
& \frac{d}{d x} v_{l l^{\prime}}^{x}=-2 i\left[v^{y}, v^{z}\right]_{l l^{\prime}}-2 n \operatorname{Tr}\left(v^{z} v^{z}\right) v_{l l^{\prime}}^{x}+4 n \operatorname{Tr}\left(v^{x} v^{z}\right) v_{l l^{\prime}}^{z} \\
& \frac{d}{d x} v_{l l^{\prime}}^{y}=-2 i\left[v^{z}, v^{x}\right]_{l l^{\prime}}-2 n \operatorname{Tr}\left(v^{z} v^{z}\right) v_{l l^{\prime}}^{y}+2 n \operatorname{Tr}\left(v^{y} v^{z}\right) v_{l l^{\prime}}^{z} \\
& \frac{d}{d x} v_{l l^{\prime}}^{z}=0 .
\end{aligned}
$$

These equations are valid in the region of $x$ where $v_{l l^{\prime}}^{x}(x), v_{l l^{\prime}}^{y}(x) \ll v_{l l^{\prime}}^{z}(0)$.

We have verified by numerical calculations that the last term in Eqs. (66) and (67) are negligible [28], although we do not present it here. Then, if we choose the representation where $v_{l l^{\prime}}^{z}$ is diagonal, i.e. $v_{l l^{\prime}}^{z}=v_{l}^{z} \delta_{l l^{\prime}}$, the scaling equations above can be solved separately with the boundary condition $v_{l l^{\prime}}^{y}(0)=0$ in the following forms

$$
\begin{aligned}
& v_{l l^{\prime}}^{x}(x)=v_{l l^{\prime}}^{x}(0) \cosh \left(2\left[v_{l^{\prime}}^{z}(0)-v_{l}^{z}(0)\right] x\right) \exp \left(-2 n \operatorname{Tr}\left[v^{z}(0) v^{z}(0)\right] x\right), \\
& v_{l l^{\prime}}^{y}(x)=i v_{l l^{\prime}}^{x}(0) \sinh \left(2\left[v_{l^{\prime}}^{z}(0)-v_{l}^{z}(0)\right] x\right) \exp \left(-2 n \operatorname{Tr}\left[v^{z}(0) v^{z}(0)\right] x\right), \\
& v_{l l^{\prime}}^{z}(x)=v_{l}^{z}(0) \delta_{l l^{\prime}} .
\end{aligned}
$$

This result indicates that the couplings $v_{l l^{\prime}}^{x}$ and $v_{l l^{\prime}}^{y}$ with the combination of $l$ and $l^{\prime}$, for which $\left|v_{l^{\prime}}^{z}(0)-v_{l}^{z}(0)\right|$ takes the largest value, increases most rapidly as $x$ increases by the renormalizaton-group step. Therefore, we are left with only the $2 \times 2$ subspace in the $3 \times 3$ space of matrices $v_{l l}$ 's; i.e, we can effectively describe the polarization degrees of freeedom of conduction electrons also by the pseudo-spin of $1 / 2$, i.e., $l=\uparrow$ and $\downarrow$, after the renormalization evolution is proceeded enough. In the subspace, the solutions can be rewritten in terms of the Pauli matix $\sigma_{l l^{\prime}}^{i}$ for conduction electron as $v_{l l^{\prime}}^{i}(x)=v^{i}(x) \sigma_{l l^{\prime}}^{i}$. Using these solutions, we can confirm that the last term in Eqs. (66) and (67) can be neglected. 
It is noed that the renormalization as above arises only if we take into account the electron-phonon (ion) coupling $V_{\hat{k} \hat{k}^{\prime}}^{x},(23)$, including at least quadratic term with respect to the ion displacement [6] because such a term gives the pseudo-spin-flip scattering with conduction electrons which is the heart of Kondo renormalization.

The $2 \times 2$ subspace is well-defined in a region $x>x_{1}$, where $x_{1}$ is determined by the conditions

$$
\begin{aligned}
& v^{x}\left(x_{1}\right)=v^{y}\left(x_{1}\right)=\frac{1}{2} v^{x}(0) \exp \left(4 v^{z}(0)\left[1-n v^{z}(0)\right] x_{1}\right) \\
& v^{z}\left(x_{1}\right)=v^{z}(0)
\end{aligned}
$$

In the region $x>x_{1}$, the pseudo-spin degrees of freedom for conduction electrons becomes explicit as the anharmonicity of ion vibrations grows. Thus our electron-phonon model can be mapped to an anisotropic two-channel Kondo model.

Next, we discuss the region $v^{x}(x)=v^{y}(x) \sim v^{z}(x)$. In this region, our scaling equations can be simplified as

$$
\begin{aligned}
& \frac{d}{d x} v^{x}=4 v^{x} v^{z}-4 n\left[\left(v^{x}\right)^{2}+\left(v^{z}\right)^{2}\right] v^{x}, \\
& \frac{d}{d x} v^{z}=4\left(v^{x}\right)^{2}\left[1-2 n v^{z}\right], \\
& \frac{d}{d x} \Delta^{x}=-4 n \Delta^{x}\left[\left(v^{x}\right)^{2}+\left(v^{z}\right)^{2}\right] .
\end{aligned}
$$

As will be shown in Appendix, the solutions of these scaling equations, (74)-(76), are given by

$$
x=-\frac{n}{4} \ln \left[v^{x}(0) v^{z}(0)\right]+\frac{1}{4 v^{z}(0)} \ln \left[\frac{4 v^{z}(0)}{v^{x}(0)}\right]+\frac{1}{8 v^{z}(0)} \ln \left[\frac{v^{z}(x)-v^{z}(0)}{v^{z}(x)+v^{z}(0)}\right],
$$

which is an implicit equation for $v^{z}(x)$ and $v^{x}(x)$ is also given in terms of $v^{z}(x)$ by the following relation

$$
v^{x}(x)=\left(\left[v^{z}(x)\right]^{2}-\left[v^{z}(0)\right]^{2} \frac{1-2 n v^{z}(x)}{1-2 n v^{z}(0)}\right)^{1 / 2} .
$$

The crossover temperature $T_{\mathrm{K}}$ is given so as the condition $v^{z}(x) / v^{z}(0) \gg 1$ is satisfied for $D^{\prime}<k_{\mathrm{B}} T_{\mathrm{K}}$, where the last term in Eq. (77) can be ignored. Then solving Eq. (77) without the last term, we obtain $T_{\mathrm{K}}$ as follows: 


$$
\bar{T}_{\mathrm{K}} \equiv \frac{k_{\mathrm{B}} T_{\mathrm{K}}}{D}=\left(\frac{v^{x}(0)}{4 v^{z}(0)}\right)^{1 / 4 v^{z}(0)}\left[v^{x}(0) v^{z}(0)\right]^{n / 4} .
$$

In the crossover region, the second order terms of the $\beta$-function, i.e., the right-hand side of the scaling equations, become comparable to that of first order. Thus the higher order terms of renormalization must be taken into account for lower energy process in gerneral. Namely, the perturbational renormalization-group theory can not be applied below $T_{\mathrm{K}}$. However, it has been known that the two-channel Kondo model has the non-trivial fixed-point which is located in the intermediate coupling regime given as

$$
v^{x *}=v^{y *}=v^{z *}=\frac{1}{2 n} .
$$

So, $v^{z}$ does not diverge even below $T_{\mathrm{K}}$ in contrast with the conventional single channel Kondo problem. In this sense, $T_{\mathrm{K}}$ gives the energy scale where the crossover from weak to intermediate coupling occurs. Around the fixed point, (80), the effectve Hamiltonian describing the low-energy physics is expected to be given by that of an isotropic two-channel Kondo model.

\section{B. Renormalization of Phonon Excitation}

The first-excited energy of ion vibrations, $\Delta^{x}$, is renormalized by the scaling equation (65): $\Delta^{x}$ softens as the renormalization process is proceeded because $\operatorname{Tr}\left(v^{y} v^{y}+v^{z} v^{z}\right)>0$. This arises from the self-energy (50) and represents the physical process of screening of spring constant of ion vibration by the conduction electrons. Such an effect causes the anharmonicity of the ion vibrations logarithmically so long as the condition $x<$ $\min \left[\ln \left(D / k_{\mathrm{B}} T\right), \ln \left(1 / \bar{\Delta}^{z}(x)\right)\right]$ is not broken, even if the bare couplings are weak.

The softening of $\Delta^{x}$ is caused mainly by the coupling with $v^{z} v^{z}$ in Eq. (65), because $v^{z}$ is the largest coupling through out the renormalization steps. So, we estimate it using the scaling equation (76) without the term $\left(v^{x}\right)^{2}$, i.e.,

$$
\frac{d}{d x} \ln \left(\Delta^{x}\right)=-4 n\left(v^{z}\right)^{2} .
$$


As will be shown in Appendix, the solution of this equation is given by

$$
\Delta^{x}(x)=\Delta^{x}(0)\left[1-2 n v^{z}(x)\right]^{1 / 2}\left(\frac{v^{x}(0)}{2 v^{z}(0)}\right)^{n v^{z}(0)} .
$$

Since the energy $\Delta^{x}(x)$ is required to exchange, or flip, the pseudo-spin dynamically, it looks as if the pseudo-spin associated with phonon degrees of freedom is quenched for the scattering with the conduction electrons with low-energy scale below $\Delta^{x}(x)$. In other words, the renormalization evolutions given by Eqs. (63)-(65) are stopped there.

\section{Scaling for Typical Cases}

Now we discuss whether or not the scaling properties, discussed in the preceding sections, are really measurable as a crossover phenomenon.

The bare couplings and phonon energy are expressed in terms of the original parameters specifying our model as follows:

$$
\begin{aligned}
& a \equiv \frac{1}{\sqrt{3}} k_{\mathrm{F}} q=\frac{1}{\sqrt{3}} \sqrt{\frac{E_{F}}{\Omega} \frac{m}{M}} \sim\left(\frac{m}{M}\right)^{1 / 4}, \\
& \frac{\Omega}{D} \sim \frac{\Omega}{E_{F}} \sim\left(\frac{m}{M}\right)^{1 / 2} \sim a^{2} \\
& b \equiv \frac{g}{D}
\end{aligned}
$$

where $E_{\mathrm{F}}$ is the Fermi energy of conduction electrons. Typical values of parameters are $a \sim 10^{-2}-10^{-1}$ and $b \sim 10^{0}$. The crossover temperature $\bar{T}_{\mathrm{K}}$, (79), and the first-excited energy $\Delta^{x}\left(T_{\mathrm{K}}\right)$, (82), of ion vibrations in the crossover region are expressed in terms of these parameters as

$$
\begin{aligned}
& \bar{T}_{\mathrm{K}}=2^{-\frac{1}{2 a b}} a^{\frac{3 n a b+1}{4 a b}} b^{\frac{n}{2}}, \\
& \Delta^{x}\left(T_{\mathrm{K}}\right)=2^{-(n a b+1)} a^{n a b+2},
\end{aligned}
$$

where we have assumed $v^{z}\left(T_{\mathrm{K}}\right) \ll 1$ in Eq. (82).

$\bar{T}_{\mathrm{K}}$ and $\Delta^{x}\left(T_{\mathrm{K}}\right)$ depend sensitively on $a$ and $b$, and their dependences are shown in Fig. 4 and 5, respectively. An anomalous behavior of physical quantities are observable for 
sufficiently large $T_{\mathrm{K}}$ and small $\Delta^{x}\left(T_{\mathrm{K}}\right)$, which are realized for appropriate value of $m / M$ and large one of $g / D$. It is because small value of $m / M$ gives extremely small $T_{\mathrm{K}}$, while large value of $m / M$ gives large $\Delta^{x}\left(T_{\mathrm{K}}\right)$ and small $g / D$ provides small $T_{\mathrm{K}}$ and large $\Delta^{x}\left(T_{\mathrm{K}}\right)$. In other words, it is preferred that the electron-phonon coupling is large and the bandwidth is small. Such situation is expected to occur in d-band metals with narrow bandwidth such as A15 and C15 compounds [2,29].

It is important to note that the region $k_{\mathrm{B}} T / D<\Delta^{x}(x)$ is not reached by the renormalization-group evolution if the parameters provide us with the crossover temperature $\bar{T}_{\mathrm{K}}<\Delta^{x}(x)$. It is because the renormalization giving divergence of coupling constants $v$ 's are cut by the fictitious magnetic field acting on the pseudo-spin at around $D^{\prime} / D=\Delta^{x}(x)$.

Typical cases of scaling are shown in Fig. 6. The case of $\bar{T}_{\mathrm{K}} \gg \Delta^{x}(x)$ is shown in Fig. 6(a), where $k_{\mathrm{B}} T / D$, the dotted line, is always larger than $\Delta^{x}(x)$, the solid line, in the right side of figure. In this case, anomalous behavior can be observed. It is noted that the coupling $v^{x}$ and $v^{y}$ increase rapidly below $T<T_{\mathrm{K}}$ while the coupling $v^{z}$ decreases reflecting the nature of two-channel Kondo effect. In the right side of figures (b) and (c), the energy scale quenching the pseudo-spin $\Delta$ determined by the intersection between the solid line, $\Delta^{x}(x)$, and the dotted line, $k_{\mathrm{B}} T / D$, gives us the energy scale where the renormalizationgroup evolution should be stopped. The case of $\bar{T}_{\mathrm{K}}<\Delta$ is shown in Fig. 6(b). The first-excited energy of ion vibration is quenched before anomalous behavior sets in. The

case of $\bar{T}_{\mathrm{K}} \ll \Delta$ is shown in Fig. 6(c). Any anomaly cannot be measurable because of extreamly small $T_{\mathrm{K}}$.

\section{CONCLUSIONS AND DISCUSSIONS}

We have discussed the structure of renormalization-group evolutions of the strongly coupled electron-phonon system. The phonon degrees of freedom have been described by the Einstein oscillator. In order to investigate the low-energy physics, the Hilbert space of phonon has been restricted so that the states with more than two phonons are prohibited 
though such states determine the algebra of the restricted phonon operators through the intermidiate or virtual states; and the conduction electrons are represented by spherical harmonics of $\mathbf{k}$ vectors on the Fermi surface because their relevant degrees of freedom is those around the Fermi level.

The simplified Hamiltonian has the pseudo-spin degrees of freedom from the beginning due to phonons in the restricted Hilbert space, while the electrons have only a latent feature of the pseudo-spin which manifests itself through the renormalization steps. Namely, the scaling equations, which is derived by the multiplicative renormalization-group method at the two-loop level, shows that the polarization of conduction electrons corresponding to those of the phonon are selectively grown at the initial stage of renormalization-gruop evolution. Thus, the effctive Hamiltonian for low-energy physics are reduced to the anisotropic twochannel Kondo model with renormalized fictitious magnetic field acting on the pseudo-spin.

In the anisotropic two-channel Kondo model with magnetic field, the scaling equations provide us with two characteristic energy scales, the crossover temperature $T_{\mathrm{K}}$ between the weak and strong coupling region and the renormalized first-excited energy $\Delta^{x}$ of phonons which is the fictitious magnetic filed acting on the pseudo-spin. The anomaly associated with two-channel Kondo effect is observable when $T_{\mathrm{K}}>\Delta^{x}$ which is realized if the mass ratio $M / m$ and the electron-phonon coupling $g / D$ are large enough. It is expected that such a sutiation is realized in the transition compounds with narrow bandwidth including A15 and C15 compounds.

The above result may be interpreted in terms of the adiabatic potential the ion feels as follows. As discussed in Refs. 2), 9), and 6), strong enough electron-phonon coupling makes the harmonic potential of individual Einstein oscillator be flattened and finally double-well shape in general. Namely, the adiabatic potential is deformed as shown schematically in Fig. 7. As a result, the first-excited energy of ion vibrations is softed considerably due to the two-channel Kondo correlation of pseudo spins.

A salient feature of the above result is that the electron-phonon coupling suffers considerable renormalization due to pseudo-Kondo effect. Namely, the so-called Migdal approxi- 
mation is not valid in this case because the higher order terms give logarithmically divergent contributions for the vertex correction. This latter situation may have been overlooked in the argument of proving a validity of the Migdal approximation. So the way that the Migdal approximation is broken down in the present theory appears to be somewhat different from that discussed by Varma [29], and Suzuki and Motizuki [30] in which the fact that the correlation length of CDW transition is very short is the origin of its breakdown.

It is well recognized that the spin susceptibility is not renormalized by the electronphonon interaction as far as the Migdal approximation is valid [24,25,29]. However, it seems still remain as a controversy whether the breakdown of the Migdal approximation directly implies the existence of renormalization of the spin susceptibility due to electron-phonon coupling [9,29, 30]. The present result suggests that only the electron-phonon coupling cannot afford to renormalize the spin susceptibility which corresponds to the channel susceptibility in our fixed-point two-channel Kondo model. In order to obtain the enhancement of channel susceptiblity in that model, we need the perturbations breaking the particle-hole symmetry, such as the Coulomb repulsion among conduction electrons or potential scattering, as shown in Ref. 21) by the method of numerical renormalization group. In this sense, together with the Coulomb repulsion among conduction electrons, the interaction (24), representing the potential scattering, may be important to discuss a possible anomaly of the (real) spin susceptibility. It is intersting to note the logarithmic temperature dependence of spin susceptiblity observed in $\mathrm{V}_{3} \mathrm{Si}$ [4] is consistent with the scenario presented in this paper. 


\section{ACKNOWLEDGEMENTS}

This work is supported by the Grant-in-Aid for Scientific Research (07640477), and Monbusho International Scientific Programs (07044078) and (06044135), and the Grant-inAid for Scientific Research on Priority Areas "Physics of Strongly Correlated Conductors" (06244104) of Ministry of Education, Science and Culture, and the Research Fellowships of the Japan Society for the Promotion of Science for Young Scientists.

\section{APPENDIX A:}

In this Appendix the solutions of equations (74)-(76) are given exactly and their simplified forms including only the sigular terms are derived for the case $v^{x}\left(x_{1}\right) \ll v^{z}\left(x_{1}\right) \ll 1$.

First, Eq. (75) is rearranged to eliminate $v^{x}$ as

$$
\left(v^{x}\right)^{2}=\frac{d v^{z} / d x}{4\left(1-2 n v^{z}\right)} .
$$

Then substituting this into Eq. (74) multiplied by $v^{x}$, we obtain

$$
\frac{1}{4} \frac{d}{d x}\left[\frac{d v^{z} / d x}{\left(1-2 n v^{z}\right)^{2}}\right]=\frac{d}{d x}\left[\frac{\left(v^{z}\right)^{2}}{1-2 n v^{z}}\right] .
$$

It is easily integrated to give the relation

$$
d x=\frac{1}{4} \frac{1}{\left(1-2 n v^{z}\right)\left[\left(v^{z}\right)^{2}+2 n C^{2} v^{z}-C^{2}\right]} d v^{z},
$$

where $C$ is determined by the boundary condition at $x=x_{1}$ as

$$
C=\left(\frac{\left[v^{z}\left(x_{1}\right)\right]^{2}-\left[v^{x}\left(x_{1}\right)\right]^{2}}{1-2 n v^{z}\left(x_{1}\right)}\right)^{1 / 2} .
$$

By integrating (A3), we obtain $v^{z}(x)$ as an implicit form:

$$
\begin{aligned}
& x-x_{1}=-\frac{n}{4} \ln \left[\frac{\left(1-2 n v^{z}\right)^{2}}{\left(v^{z}\right)^{2}+2 n C^{2} v^{z}-C^{2}} \cdot \frac{\left[v^{z}\left(x_{1}\right)\right]^{2}+2 n C^{2} v^{z}\left(x_{1}\right)-C^{2}}{\left[1-2 n v^{z}\left(x_{1}\right)\right]^{2}}\right] \\
& +\frac{2 n^{2} C^{2}+1}{8 C \sqrt{n^{2} C^{2}+1}} \ln \left[\frac{v^{z}+n C^{2}-C \sqrt{n^{2} C^{2}+1}}{v^{z}+n C^{2}+C \sqrt{n^{2} C^{2}+1}} \cdot \frac{v^{z}\left(x_{1}\right)+n C^{2}+C \sqrt{n^{2} C^{2}+1}}{v^{z}\left(x_{1}\right)+n C^{2}-C \sqrt{n^{2} C^{2}+1}}\right] .
\end{aligned}
$$


For the case $v^{x}\left(x_{1}\right) \ll v^{z}\left(x_{1}\right) \sim v^{z}(0) \ll 1$, keeping only the singular terms in right-hand side of Eq. (A5), we obtain

$x-x_{1}=-\frac{n}{2} \ln \left[v^{x}\left(x_{1}\right)\right]-\frac{n}{4} \ln \left[\frac{2 v^{z}(0)}{v^{x}\left(x_{1}\right)}\right]+\frac{1}{4 v^{z}(0)} \ln \left[\frac{2 v^{z}(0)}{v^{x}\left(x_{1}\right)}\right]+\frac{1}{8 v^{z}(0)} \ln \left[\frac{v^{z}-v^{z}(0)}{v^{z}+v^{z}(0)}\right]$.

We can also obtain the explict form for $x_{1}$ from Eq. (72) as

$$
x_{1}=\frac{1}{4 v^{z}(0)\left[1-n v^{z}(0)\right]} \ln \left[\frac{2 v^{x}\left(x_{1}\right)}{v^{x}(0)}\right] \sim\left[\frac{1}{4 v^{z}(0)}+\frac{n}{4}\right] \ln \left[\frac{2 v^{x}\left(x_{1}\right)}{v^{x}(0)}\right] .
$$

Then, eliminating $x_{1}$ from Eqs. (A6) and (A7), we obtain Eq. (77) for $v^{z}(x)$, and then we also obtain Eq. (78) for $v^{x}(x)$ from Eqs. (A1), (A3) and (A4).

Second, we eliminate $d x$ from Eq. (81) by using Eq. (A3). Then, the obtained equation can be integrated with the boundary condition at $x=0$ as follows:

$$
\begin{aligned}
& \frac{\Delta^{x}(x)}{\Delta^{x}(0)}=\left(\frac{1-2 n v^{z}}{1-2 n v^{z}(0)}\right)^{1 / 2} \times \\
& \quad \times\left(\frac{v^{z}+n C_{0}^{2}+C_{0} \sqrt{n^{2} C_{0}^{2}+1}}{v^{z}+n C_{0}^{2}-C_{0} \sqrt{n^{2} C_{0}^{2}+1}} \cdot \frac{v^{z}(0)+n C_{0}^{2}-C_{0} \sqrt{n^{2} C_{0}^{2}+1}}{v^{z}(0)+n C_{0}^{2}+C_{0} \sqrt{n^{2} C_{0}^{2}+1}}\right)^{n C_{0} / 2 \sqrt{n^{2} C_{0}^{2}+1}},
\end{aligned}
$$

where $C_{0}$ is given by substituting $v^{x}\left(x_{1}\right)$ and $v^{z}\left(x_{1}\right)$ by $v^{x}(0)$ and $v^{z}(0)$, respectively, in Eq. (A4). For the case $v^{x}(0) \ll v^{z}(0) \ll 1$, keeping only the singular terms of the original couplings, we obtain the Eq. (82). 


\section{REFERENCES}

[1] M. Weger and I. B. Goldberg: Solid State Physics, eds. H. Ehrenreich, F. Seitz and D. Turnbull (Academic Press, New York and London) 28 (1973) 1.; L. R. Testrardi: Rev. Mod. Phys. 47 (1975) 637.

[2] P. W. Anderson and C. C. Yu: Proc. Int. School of Physics "Enrico Fermi" eds. F. Bassani et al (North-Holland, Amsterdam, 1985), p. 767.

[3] G. R. Hearne, P. R. Stoddart and H. Pollak: Physica C 167 (1990) 415.

[4] J. P. Maita and E. Bucher: Phys. Rev. Lett. 29 (1972) 931.

[5] T. Ohno, Y. Kishimoto, H. Kotaki, T. Yamanishi, T. Kanashiro, Y. Michihiro and Y. Yamada: Physica B 186-188 (1993) 1034.

[6] T. Matsuura and K. Miyake: J. Phys. Soc. Jpn. 55 (1986) 29; 610.

[7] K. Miyake, T. Matsuura and C. M. Varma: Solid State Commun. 71 (1989) 1149.

[8] M. D. Núñez-Regueiro, J. M. Lopez-Castillo and C. Ayache: Phys. Rev. Lett. 55 (1985) 1931.

[9] C. C. Yu and P. W. Anderson: Phys. Rev. B29 (1984) 6165.

[10] K. Vladár and A. Zawadowski: Phys. Rev. B28 (1983) 1564; 1582; 1596.

[11] P. Nozières and A. Blandin: J. Phys. (Paris) 41 (1980) 193.

[12] D. L. Cox: Phys. Rev. Lett. 59 (1987) 1240; J. Magn. \& Magn. Matter 76 \& 77 (1988) 53.

[13] A. Muramatsu and F. Guinea: Phys. Rev. Lett. 57 (1986) 2337.

[14] D. M. Cragg, P. Lloyd and P. Nozières: J. Phys. C13 (1980) 803.

[15] H. B. Pang and D. L. Cox: Phys. Rev. B 44 (1991) 9454. 
[16] O. Sakai, Y. Shimizu and N. Kaneko: Physica B 186-188 (1993) 323.

[17] N. Andrei and C. Destri: Phys. Rev. Lett. 52 (1984) 364.

[18] A. M. Tsvelik and P. B. Wiegmann: J. Stat. Phys. 38 (1985) 125.

[19] V. J. Emery and S. Kivelson: Phys. Rev. B 46 (1992) 10812.

[20] P. Coleman, L. Ioffe and A. M. Tsvelik: preprint cond-mat/9504006.

[21] H. Kusunose, K. Miyake, Y. Shimizu and O. Sakai: Phys. Rev. Lett. 76 (1996) 271.

[22] N. Menyhárd and J. Sólyom: J. Low. Temp. Phys. 12 (1973) 529.

[23] J. Sólyom: J. Phys. F 4 (1974) 2269.

[24] J. J. Quin: The Fermi Surface eds. W. A. Harrison and M. B. Webb (Academic Press, New York, 1955), p. 58.

[25] R. E. Prange and L. P. Kadanoff: Phys. Rev. 134 (1964) A566.

[26] J. Kondo: Prog. Theor. Phys. 32 (1964) 37.

[27] A. A. Abrikosov: Physics 2 (1965) 5.

[28] These terms are neglected in Ref. 21 due to the assumption $v^{y}, v^{z} \ll v^{x}$. This assumption, however, is not valid, thus it is not trivial if these term can be neglected or not with no calculation.

[29] C. M. Varma: Charge Density Waves in Solids eds. Gy. Hutirary and J. Sólyom (Springer, Berlin, 1985), p. 99, and references therein.

[30] N. Suzuki and K. Motizuki: Structural Phase Transitions in Layered Transition-Metal Compounds ed. K. Motizuki (D. Reidel Publishing Company, Dordrecht, 1986), p.135. 


\section{Figure Captions}

Fig. 1

Diagrams for vertex correction of first order. Solid (dotted) line stands for conduction electron (psudo-spin).

Fig. 2

Diagrams for vertex correction of second order.

Fig. 3

Diagram for Self-energy of pseudo-fermion of first order.

Fig. 4

$(m / M)^{1 / 4}$ dependence of $\bar{T}_{\mathrm{K}}$ and $\Delta^{x}\left(T_{\mathrm{K}}\right)$.

Fig. 5

$g / D$ dependence of $\bar{T}_{\mathrm{K}}$ and $\Delta^{x}\left(T_{\mathrm{K}}\right)$.

Fig. 6

Typical cases of scaling; (a) anomalous behavior can be observed, (b) the first-excited energy of ion vibration is quenched before anomalous behavior sets in, (c) $T_{\mathrm{K}}$ is extremely small.

Fig. 7

Schematic shape of adiabatic potential of ion; (a) for the case without electron-phonon coupling or the high-energy processes, and (b) for the fixed-point Hamiltonian or the low-energy-processes. 
Fig. 1(a)

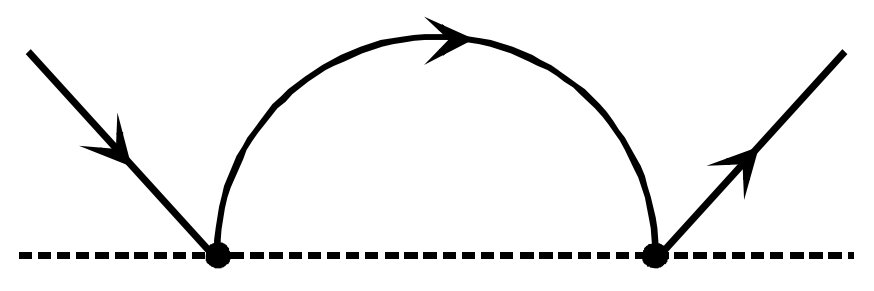

Fig. 1(b)

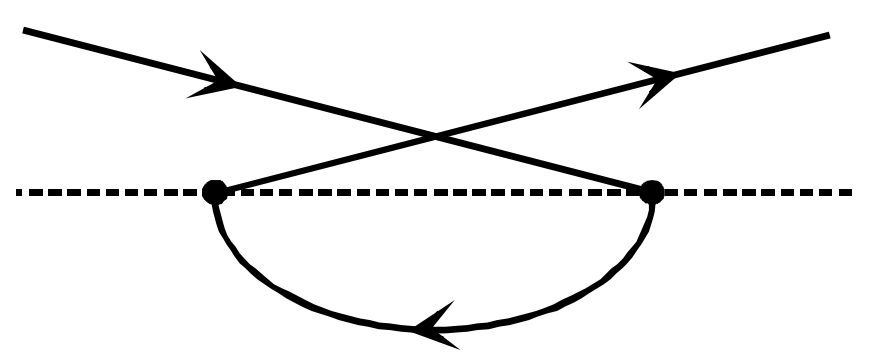

Fig. 2

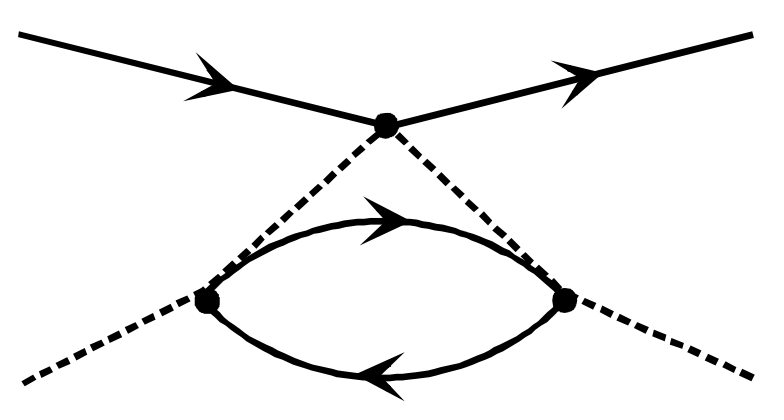

Fig. 3

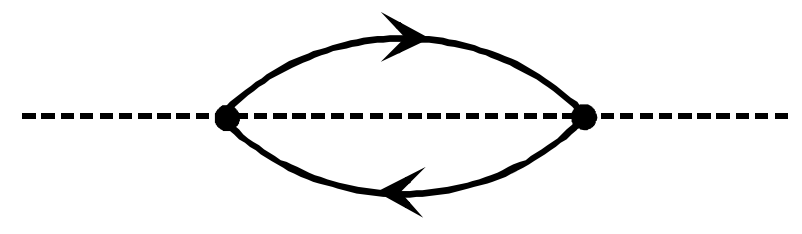

H. Kusunose and K. Miyake 
Fig. 4 H. Kusunose and K. Miyake

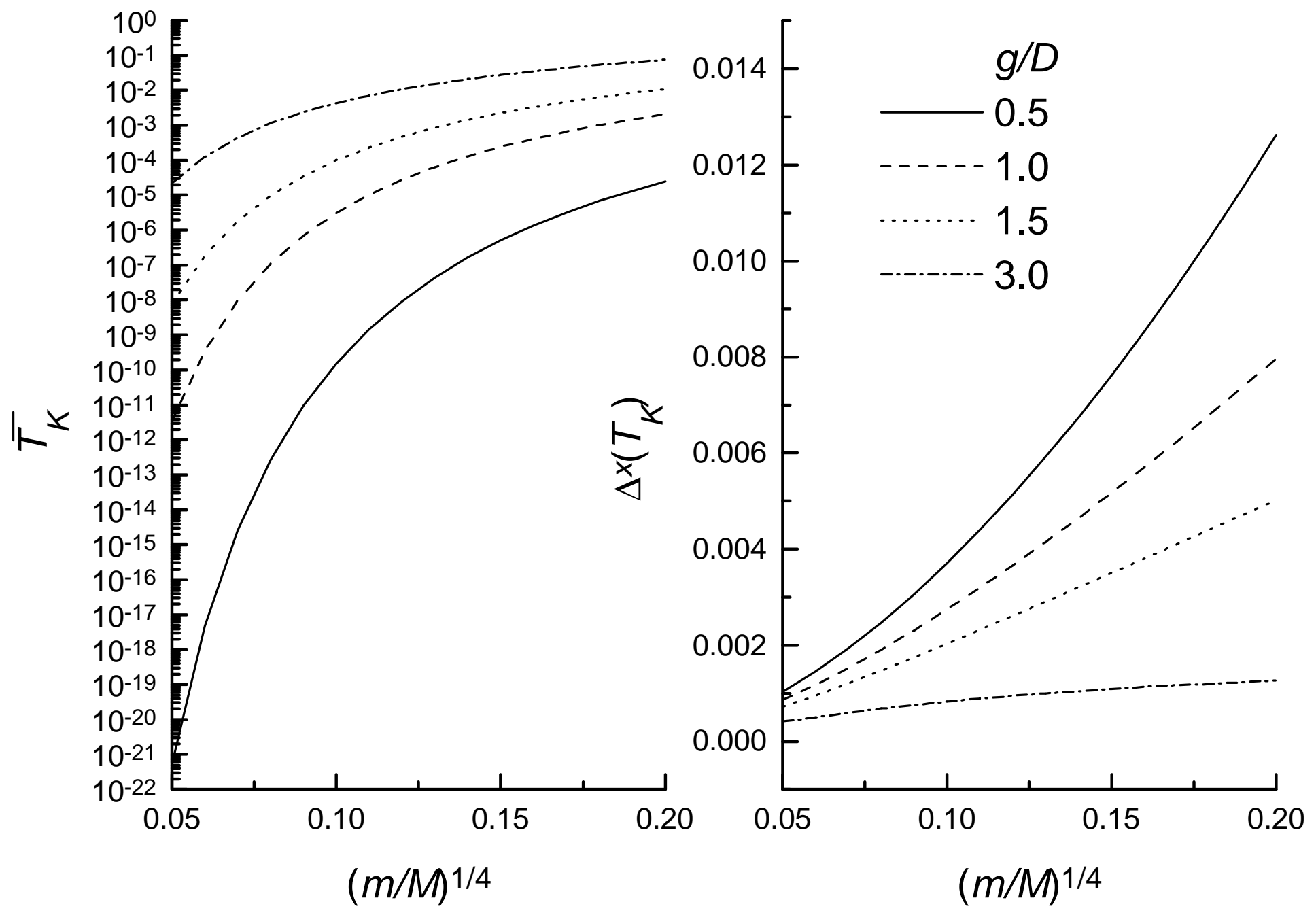


Fig. 5 H. Kusunose and K. Miyake

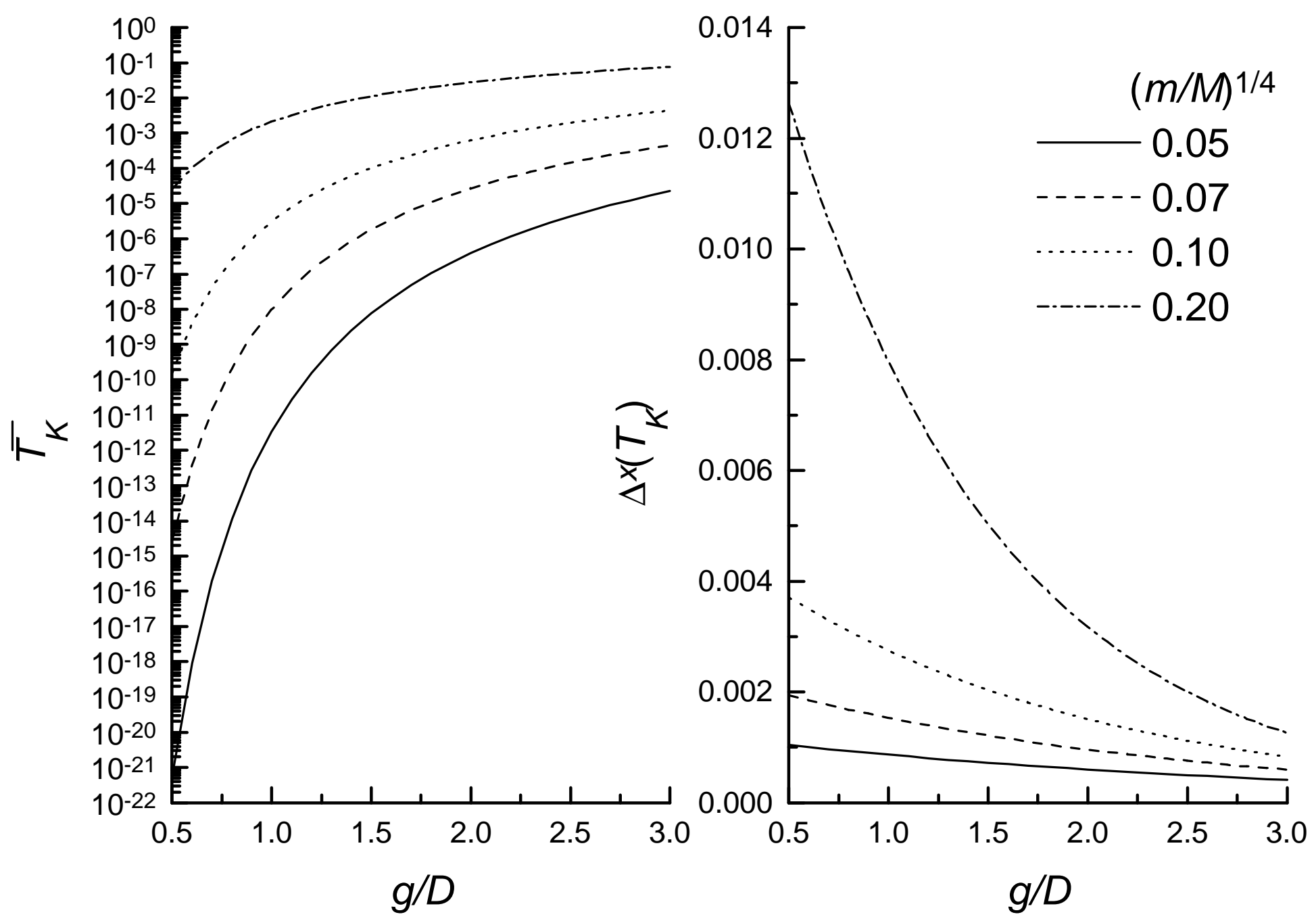


Fig. 6 (a) H. Kusunose and K. Miyake
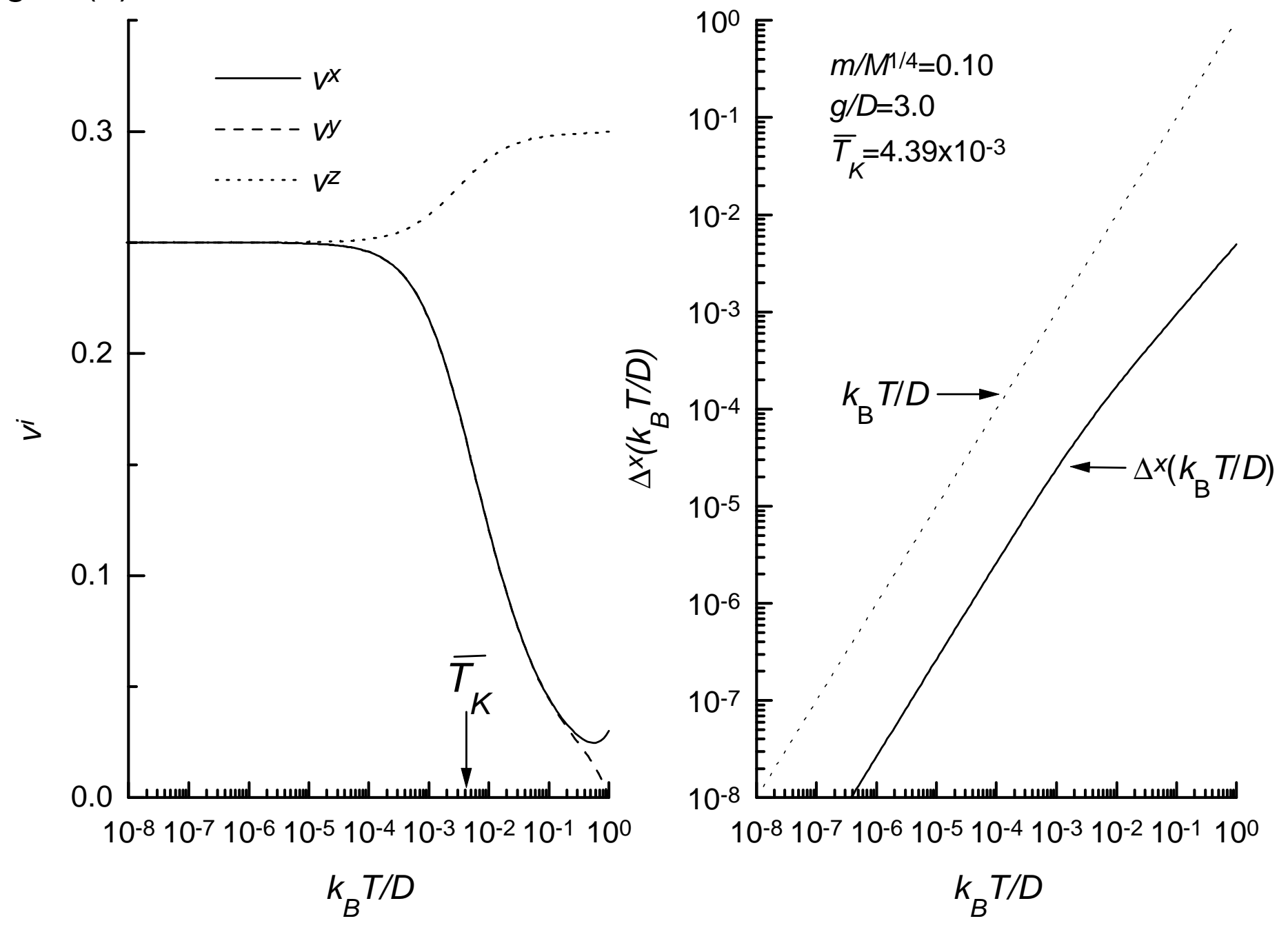
Fig. 6 (b) H. Kusunose and K. Miyake

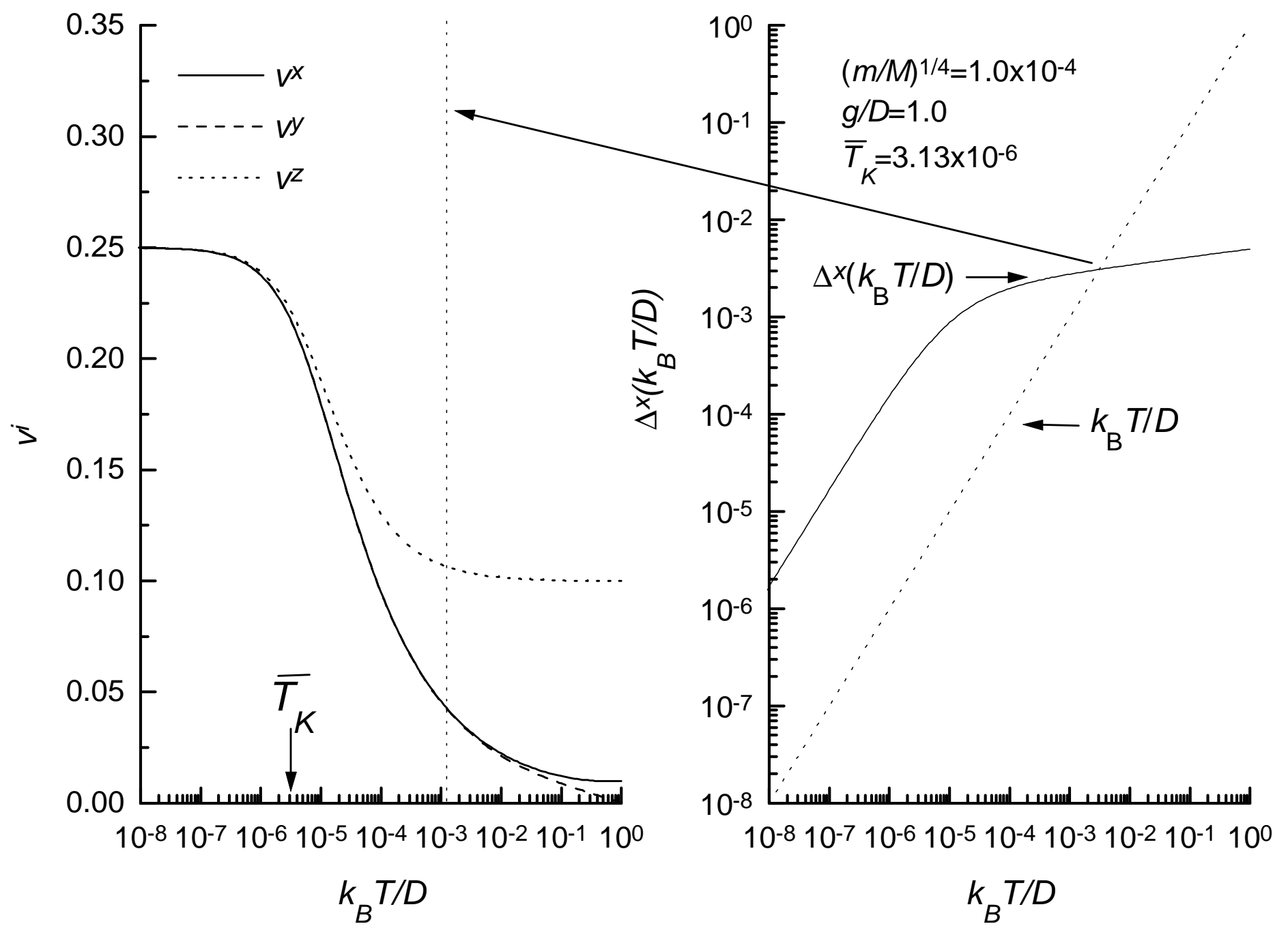


Fig. 6 (c) H. Kusunose and K. Miyake
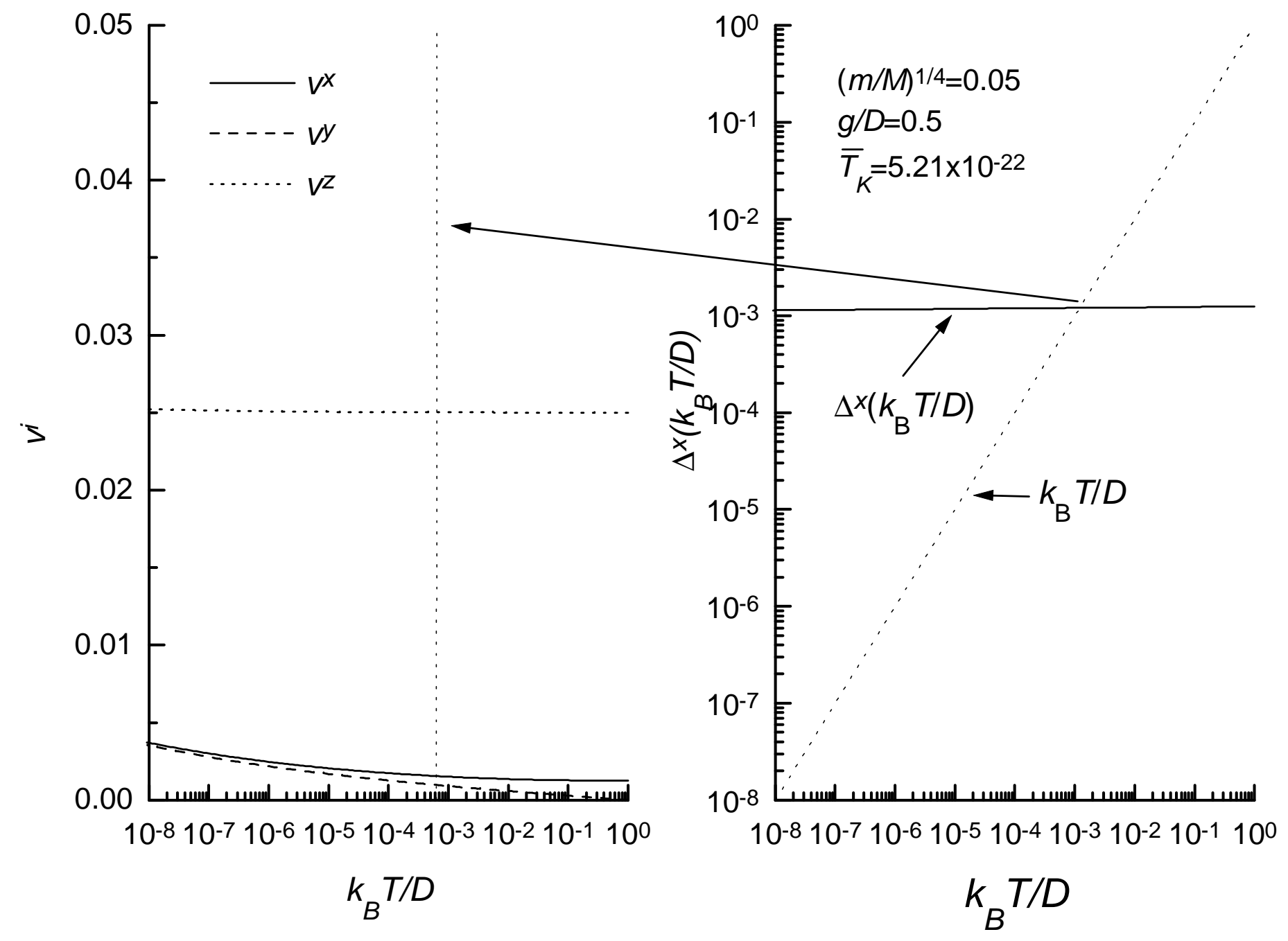
Fig. 7
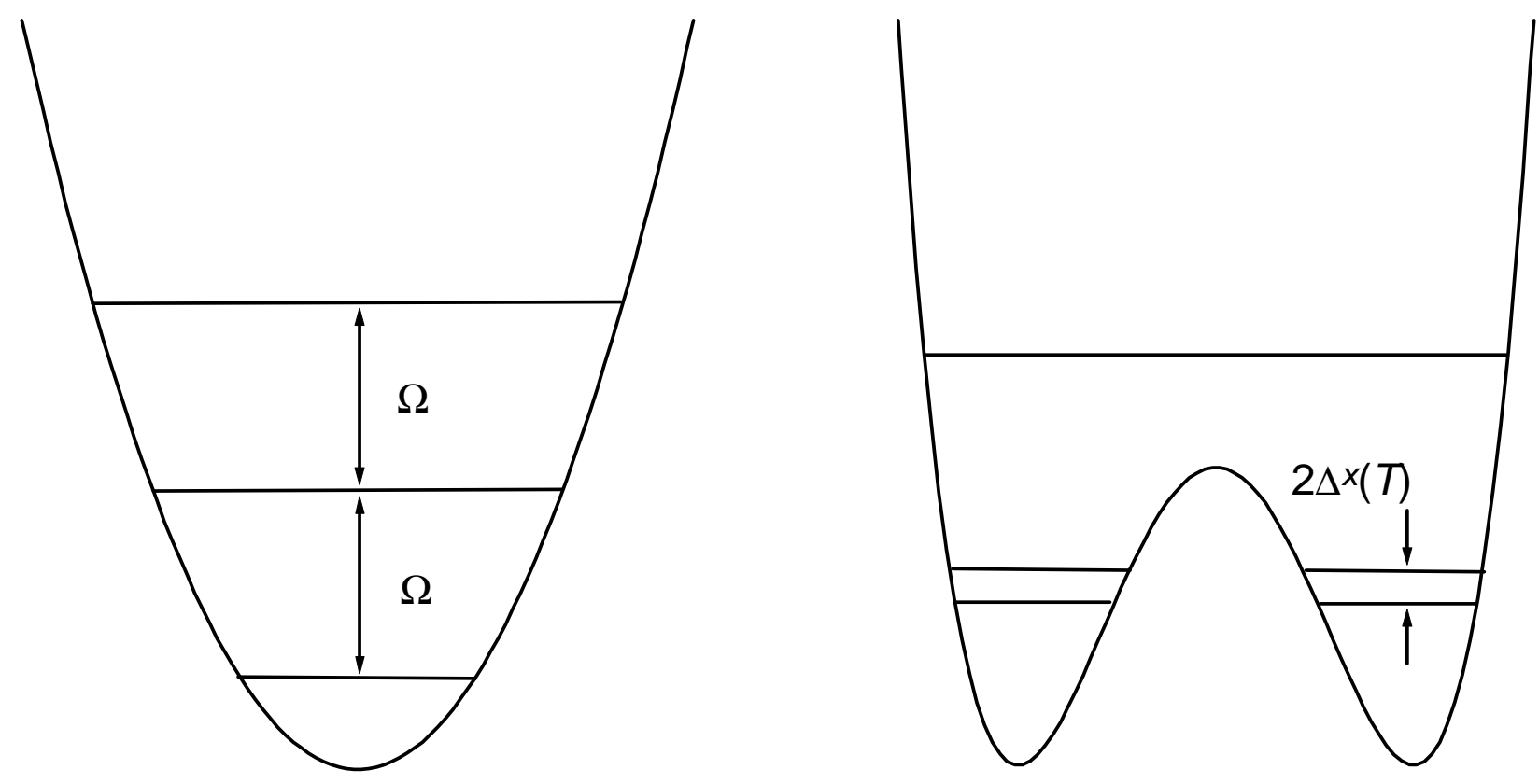

H. Kusunose and K. Miyake 